\title{
Extractos atribuidos a Virgilio y el canto XIII de La Eneida en los florilegios latinos del siglo XVI
}

Ma Elena Curbelo Tavío - Ma Dolores García de Paso Carrasco (University of Las Palmas de Gran Canaria)

\section{Excerpts Attributed to Virgil and The Aeneid' XIII Book in $16^{\text {th }}$-Century Latin Florilegia}

\begin{abstract}
Throughout the different ages, several texts attributed to Virgil have circulated apart from his three major works. Some of them are poems included in the Appendix Vergiliana; others are collected in the Carmina XII sapientum; and we even find some anecdotes that Donatus offers in the poet's life. In this work, we examine a selection of extracts collected in three $16^{\text {th }}$-century florilegia: the Illustrium poetarum flores (Strasbourg 1538), by O. Mirandula; the Sententiae ueterum poetarum (Antwerp 1541), by G. Maior; and the Versus Sententiosi et eximii (Wittenberg 1565), by B. Schönborn. We also deal with several fragments of the Libri XIII Aeneidos Supplementum by Maffeo Vegio, which is reproduced in Mirandola's compilation. The presence of texts linked to Virgil in the florilegia could indicate that the prestige he enjoyed in the Middle Ages extended into the Renaissance. In addition, in G. Maior's work there are doubts about the authorship of the texts presenting a moral and pedagogical tone, implying that moral and didactic interests may have motivated the selection.
\end{abstract}

\section{Keywords}

Appendix Vergiliana; Carmina XII sapientum; florilegia; Maffeo Vegio; Virgil

Este trabajo se enmarca dentro del Proyecto de Investigación ProlD2017010015 y la Red de Excelencia Europa Renascens. Biblioteca Digital de Humanismo y Tradición Clásica (España y Portugal) FFI2015-69200-REDT, financiados por el Gobierno de España. 


\section{Introducción}

En este artículo examinamos extractos que, sin ser de Virgilio ni formar parte del Appendix Vergiliana, circularon bajo su nombre y aparecen recogidos en tres florilegios del siglo XVI: ${ }^{1}$ Illustrium poetarum flores per Octauianum Mirandulam collecti et a studioso quodam in locos comunes nuper digesti ac castigati (Estrasburgo 1538); Sententiae ueterum poetarum per locos comunes digestae. Collectore Ioanne Maiore (Amberes 1541); y Versus Sententiosi et eximii iuxta litterarum ordinem e ueteribus poetis consignati a Bartolemeo Schonborn Witebergensi (Wittenberg 1565). ${ }^{2}$ Además, incluimos los fragmentos que aparecen en Mirandula del Libri XIII Aeneidos Supplementum (Pavía 1428), de Maffeo Vegio.

Los Illustrium poetarum flores es una nueva presentación del Viridarium illustrium poetarum cum ipsorum concordantiis in alphabetica tabula accuratissime contentis (Venecia 1507) de O. Mirandula ${ }^{3}$ realizada por un escoliasta anónimo y que ofrece los excerpta distribuidos por lemas o tituli (loci communes), que siguen un orden alfabético, y sublemas. ${ }^{4}$

Las Sententiae ueterum poetarum de G. Maior (1534) también presentan los extractos organizados bajo lemas. Del éxito alcanzado por este florilegio dan fe las numerosas ediciones realizadas desde su composición y hasta el año 1600 (Artigas et al. 2014: pp. 965-967; Green \& Murphy 2006: p. 286). La edición publicada en Amberes en 1541 es la que hemos empleado como corpus de estudio.

Los Versus Sententiosi et eximii de B. Schönborn (Wittenberg 1564) no presentan lemas, sino que los excerpta se ofrecen en orden alfabético en una clara imitación de las recopilaciones de sentencias y glosarios de la Antigüedad (Olsen 1979: pp. 47-53; Artigas et al. 2014: p. 928). Será por la edición de este florilegio realizada en 1565 por la que citaremos. ${ }^{5}$

1 Nuestro corpus de estudio se ha extraído de la base de datos del Proyecto Excerpta (http://excerpta.iatext. ulpgc.es), coordinado por Gregorio Rodríguez Herrera y liderado por el Instituto Universitario de Análisis y Aplicaciones Textuales de la Universidad de Las Palmas de Gran Canaria (Rodríguez Herrera \& Rodríguez Rodríguez \& Santana Jaria 2020).

2 Los florilegios Ex Elegiis Tibulli, Propertii et Ouidii ab Ioanne Murmellio Selecti uersus (París 1533 /=Breda 1504/) y las Sententiae et prouerbia ex poetis latinis (ab Roberto Stephano) - His adiecimus Leosthenis Coluandri sententias prophanas (Venecia 1547 /=París 1534/), recogidos en el Proyecto Excerpta, se han excluido de nuestro análisis por no contener extractos de Virgilio el primero y por carecer de excerpta del Appendix y de los otros poemas atribuidos erróneamente a Virgilio el segundo.

3 Artigas et al. (2014: p. 956) recogen 4 ediciones de este florilegio: Lyon (1512), dos en París (1513) y Haguenau (1517). Los Illustrium poetarum flores, en cambio, fueron reeditados, al menos, treinta y ocho veces entre 1538 y 1600 (Artigas et al. 2014: p. 958).

4 Varios trabajos han intentado ofrecer una clasificación de los florilegios atendiendo a factores como los autores que se extractan, las obras, su contenido o la finalidad que persiguen. Entre ellos destacan Olsen (1979: pp. 47-121; 1980: pp. 115-160); Fernández de la Cuesta (2008: pp. 50-54).

5 En Wittemberg, donde este florilegio fue editado por vez primera, se realizaron 10 ediciones entre 1564 y 1610 . 


\section{Virgilio y el Appendix Vergiliana en nuestro corpus de estudio}

La presencia de Virgilio en los florilegios, exceptuado el caso de Schönborn, no es lo abundante que cabría esperar, dada la gran fama de la que goza el poeta. ${ }^{6}$ A las referencias a las tres grandes obras del Mantuano hay que añadir las citas pertenecientes a una serie de poemas que se recogen actualmente bajo la denominación de Appendix Vergiliana ${ }^{7}$ y que se consideran trabajos juveniles de Virgilio, supuestamente compuestos antes de las Eclogae. Esta colección coincide, en gran medida, con las obras mencionadas por Suetonio, Elio Donato ${ }^{8}$ y Servio, ${ }^{9}$ es decir: Catalepton, Priapea, Epigrammata, Dirae (que algunos dividen en dos, Dirae y Lydia), los epilios Ciris y Culex, Aetna y Copa. A las anteriores se añaden Moretum y las Elegiae in Maecenatem, dadas a conocer por un catálogo del s. IX de la Biblioteca de Murbach (Recio \& Soler 1990: p. 398), ${ }^{10}$ y en muchas ediciones se incluyen también tres obras atribuidas a Ausonio, De rosis nascentibus, Est et non y Vir bonus (Moya 1982: p. 409).

En los florilegios estudiados hay grandes diferencias en cuanto a los extractos atribuidos a Virgilio. De los poemas recopilados por el Appendix, en Mirandula aparecen un total de 17 extractos de los siguientes poemas: 9 de Aetna, 2 de Ciris, 1 de Dirae, 1 de Est et non y 4 de Elegiae in Maecenatem. En Maior y Schönborn, sin embargo, no hay fragmentos del Appendix.

Un caso particular es Vir bonus pues, aunque se atribuye a Ausonio, en muchas ediciones se incluye en el Appendix (Moya 1982: p. 409). Pues bien, en Maior hay un excerptum perteneciente a Vir bonus y en Mirandula, cuatro. El extracto de Maior ofrece el poema completo y tiene como lema Boni uiri exemplum. El lema de tres de los cuatro extractos de Mirandula es De bonitate y en ellos se encuentra el poema fragmentado: uno de los fragmentos incluye los vv. 1-2; otro, los vv. 3-13 y el tercero, los vv. 14-26. Por otro lado, el cuarto fragmento lleva por lema De electis y comprende los vv. 1-2.

6 Los extractos de Virgilio suman aproximadamente un 2\% del total de extractos del florilegio de Maior, un $3 \%$ de los de Mirandula y un 30\% de los de Schönborn. Para la presencia de Virgilio en estos florilegios, v. Curbelo Tavío \& García de Paso (2020).

7 Recio \& Soler (1990: p. 397) indican que, aunque la extensión de este nombre se debe a Escalígero, que publicó en las prensas de Gulielmus Rouillius en París (1573) una edición comentada que tituló Publii Virgilii Maronis Appendix cum supplemento multorum antehac nunquam excusorum Poematum ueterum poetarum Iosephi Scaligeri in eandem Appendicem commentarij $\mathcal{E}$ castigationes, ya en 1521 Valeriano, comentando las Bucólicas, había señalado: ut in appendicibus Virgilii legitur.

8 Puer [...] "monte sub hoc lapidum tegitur Ballista sepultus; nocte die tutum carpe uiator iter", deinde Catalepton et Priapea et Epigrammata et Diras, item Cirim et Culicem cum esset annorum XVI [...] scripsit etiam de qua ambigitur Aetnam. Mox cum res Romanas incohasset, offensus materia ad Bucolica transiit [...] (Brummer 1933: pp. $4-5)$.

9 Primum ab hoc distichon factum est in Ballistam latronem "Monte sub hoc lapidum tegitur Ballista sepultus: / nocte die tutum carpe uiator iter." Scripsit etiam septem siue octo libros hos: Cirim, Aetnam, Culicem, Priapeia, Catalepton, Epigrammata, Copam, Diras. Postea ortis bellis ciuilibus inter Antonium et Augustum [...] (Brummer 1933: p. 69).

10 Los textos que se encuentran incluidos en un catálogo del s. IX del monasterio de Murbach y que fueron transcritos por Meisterlin (1464) son: Dirae, Culex, Aetna, Copa, Elegiae in Maecenatem, Ciris, Priapea et Catalepton, Priapeum "Quid hoc noui est", Moretum, De institutione uiri boni, De est et non, De rosis nascentibus (Schniebs et al. 2014: p. 11). 


\section{Otras composiciones atribuidas a Virgilio en nuestro corpus de estudio}

Los poemas del Appendix no son los únicos que circulaban bajo el nombre de Virgilio, pues se le atribuyeron otros muchos. En nuestro corpus encontramos fragmentos de ocho de ellos: De Fortuna, De littera Pythagorica, De Liuore, De signis coelestibus, De uino et Venere, De Orpheo, De aetatibus animantium y De Musarum inuentis. Algunos están incluidos en los Carmina XII sapientum, una obra de la Antigüedad tardía y atribuida a Lactancio, ${ }^{11}$ que se difundió en la Edad Media y el Renacimiento. ${ }^{12}$

Los Carmina o Symposium duodecim sapientum han sido calificados como 'enciclopedia epigramática’, pues destaca su propósito educativo tanto por la temática como por los aspectos formales (Mondin 2019: p. 580; 2016: pp. 193-198). Ofrece la forma de un certamen poético entre doce autores ficticios y consta de doce apartados, los once primeros están sometidos a unas reglas específicas respecto a la temática, al metro (hexámetros o dísticos elegíacos) y a la longitud; en cambio, el apartado doce es libre. En nuestro corpus están recogidos cinco de los poemas del apartado XII: De Fortuna, De littera Pythagorica, De Liuore, De uino et Venere, De Orpheo y uno de los poemas del apartado XI que se dedica a los doce signos del Zodíaco.

Por otro lado, en muchas de las ediciones de Virgilio aparecen, además del Appendix, otras obras menores atribuidas a él. Así, en la edición parisina de Joannes Parvus (1507), junto a las 3 obras mayores Aeneis, Georgica y Eclogae (Opera) y a Dirae, Culex, Aetna, Copa, Elegiae in Maecenatem, Ciris y Moretum (Opuscula) se incluyen otros poemas bajo el título de Epigrammata, alguno ya presente en Carmina XII sapientum. Entre los Epigrammata se encuentran Vir bonus, De Liuore, De Venere et uino, De littera Pythagorica, De aetatibus animantium, De Musarum inuentis, De Fortuna, De Orpheo y De figuris coelestibus (Kallendorf 2018: pp. 149-151).

Sin contar con el Appendix ni con Vir bonus, al que nos hemos referido en el apartado anterior, en los Illustrium poetarum flores de Mirandula hay presencia de seis de estos poemas: De Fortuna, De littera Pythagorica, De Liuore, De signis coelestibus, De Orpheo y tres excerpta de De uino et Venere; y en el florilegio de Maior, siete: De aetatibus animantium, De Fortuna, De littera Pythagorica, De Liuore, Musarum inuenta, Duodecim signa coelestia y De uino et Venere. Finalmente, en los Versus sententiosi de Schönborn se incluyen, bajo el título de Epig. (Epigrammata), cinco fragmentos atribuidos a Virgilio que se corresponden con un solo poema, De uino et Venere.

En las aulas, durante la Edad Media y el Renacimiento, Virgilio parece haber sido estudiado por el carácter moral que se le atribuía y que se recoge no solo en los discursos de La Eneida, sino en los proverbios y aforismos, fáciles de recordar, así como por el correcto uso del lenguaje de la obra, pues ambos aspectos lo hacían apto para su utilización en los ejercicios retóricos (Kallendorf 2013: p. 318). Ello justifica, también,

11 Esto conllevaría adelantar el renacimiento de la poesía epigramática latina, generalmente datado en la segunda mitad del s. IV (Mondin 2019: p. 578).

12 Para esta obra puede consultarse Friedrich (2002), quien ofrece un comentario detallado que aborda aspectos textuales, lingüísticos y estilísticos. 
la presencia, en los florilegios, de numerosos extractos tomados de La Eneida (Curbelo Tavío \& García de Paso 2020).

Los poemas que circulan bajo el nombre de Virgilio se caracterizan igualmente por su tono moralizante o por ciertos aspectos formales que explican su inclusión en los florilegios.

El poema De uino et Venere ${ }^{13}$ es el único que se encuentra en los tres compiladores. En él, amor y vino se asimilan, ambos son nocivos, debilitan las fuerzas, impiden guardar secretos y provocan peleas, como demuestran los exempla mythologica que se incluyen; además, eliminan el pudor, el miedo y la honradez. Por ello, hay que ser moderado y solo han de tener cabida dentro de los márgenes de lo socialmente aceptable.

Maior reproduce el poema completo, pero ignora el tema de Venus para centrarse en el vino, como muestra el lema bajo el que se incluye: Vinum, ebrietas. También Mirandula reproduce el poema completo, pero bajo el lema De abstinentia y con Abstinere a uini potatione superflua et Venere omnes debent como sublema, incidiendo, por tanto, en la necesidad de la moderación. Incluye, además, otros dos versos del mismo poema, Arcanum demens detegit ebrietas y Bellum saepe petit ferus exitiale Cupido, a modo de sententiae bajo distintos lemas: De ebrietate (sublema: Ebrietas ubi adest, ibi secretum non est), el primero y De amore (sublema: Amor mulierum inmoderatus bellorum saepe causa extitit), el segundo. En Mirandula, por tanto, a diferencia de Maior, hay equilibrio entre los males de la ebriedad (incapacidad de retener secretos) y el erotismo (causa de reyertas) y en el lema y el sublema bajo los que se ofrece el poema completo hay una llamada a la abstinencia.

De los cinco excerpta de Schönborn, coincide totalmente con Mirandula el que recoge la incapacidad del ebrio para guardar secretos: Arcanum demens detegit ebrietas. En otro, la coincidencia es parcial pues al verso Bellum saepe petit ferus exitiale Cupido se añade el siguiente, Saepe manus itidem Bacchus ad arma uocat, con lo que el alemán iguala la capacidad del amor y el vino para promover altercados.

A través de los extractos recopilados por Schönborn, se recoge perfectamente el contenido del poema. Así, uno de ellos señala que igual de dañinos son el vino y amor, por lo que hay que evitar que uno y otro nos posean: Nec Veneris, nec tu uini capiaris amore,/ Vno namque modo uina Venusque nocent. Y también ambos debilitan las fuerzas: Vt Venus eneruat uires, sic copia Bachi/ Attenuat gressus debilitatque pedes. Por último, hay una llamada a la moderación, que el vino aplaque la sed y Venus sirva para crear hijos, pues traspasar estos límites es dañino: Vina sitim sedent: natis Venus alma creandis/Seruiat: hos fines transiliisse nocet.

Los tópicos del carácter nocivo de la ebriedad y del amor ${ }^{14}$ están presentes en la literatura desde antiguo, como podemos ver en la comedia griega, en la que se pone

13 Nec Veneris, nec tu Vini tenearis amore,/ Vno namque modo Vina, Venusque nocent./ Vt Venus eneruat uires, sic copia Bacchi,/ Attenuat gressus, debilitatque pedes./ Multos caecus amor cogit secreta fateri, Arcanum demens detegit ebrietas./ Bellum saepe parit ferus exitiale Cupido,/Saepe manus itidem Bacchus ad arma uocat./ Perdidit horrendo Troiam Venus improba bello,/ Et Lapithas bello perdis lacche graui./ Denique cum menteis hominum furiarit uterque,/ Et pudor, et probitas, et metus omnis abest. Compedibus Venerem, uinclis constringe Lyaeum,/ Ne te muneribus laedat uterque suis./Vina sitim sedent, natis Venus alma creandis/ Seruiat, hos fines transiliisse nocet (Poetae latini minores, ed. Baehrens 1881: pp. 150-151).

14 Sobre los topica amatorios y las consecuencias del amor, $v$. Moreno (2011). 
a menudo de manifiesto la afición de los personajes al vino y las consecuencias de su uso desmesurado (Calderón 1999; García Soler 2010; Thiercy 1997: p. 173). El poema ofrece dos exempla mythologica ${ }^{15}$ ilustrando los efectos perniciosos de ambos. Conocida es la brutalidad de los centauros cuando beben vino (García Gual 2003: p. 93). Y, sin duda, el más significativo de los exempla mitológicos del daño que ocasiona Venus es el de la guerra de Troya, desencadenada por el amor a Helena.

Este poema no es la única coincidencia dentro de los florilegios, pues en dos de ellos, el de Maior y el de Mirandula, se encuentran estos 4 poemas: De littera Pythagorica, De Liuore, De Fortuna y De signis coelestibus.

De littera Pythagorica ${ }^{16}$ es un poema dedicado a la letra ' $\mathrm{Y}$ ', llamada letra pitagórica, que se considera una imagen de la vida humana a la que se ofrecen dos caminos, uno es muy arduo, el de la virtud, pero al final, tras el esfuerzo, se consigue la alabanza; el otro es el del vicio, en principio fácil, pero que conduce a una vida miserable. ${ }^{17}$ Tanto Maior como Mirandula lo incluyen bajo el lema de la virtud. El sublema en Mirandula explicita el contenido del fragmento: Per Pythagorae literam uirtutis ac uoluptatis uia ostenditur.

En el poema De Liuore $^{18}$ se describe la personificación de la envidia o sus efectos, como señala el sublema de Mirandula (Inuidiae descriptio, seu effectus ipsius expressio propriissi$m a)$ : produce no solo lengua venenosa, sino otros síntomas muy similares a los producidos por el furor amoris ${ }^{19}$ como sudor frío, palidez, locura e incapacidad de disfrutar de la comida, la bebida y el sueño, es más, la envidia, es una herida incurable, semejante a la que sufre Dido por su amor a Eneas (Verg. Aen. 4, 1-2, 66-67; Ov. epist. 7, 190). Los lemas bajo los que se incluye son De inuidia en Mirandula y en Maior, Inuidia, liuor, odium. La envidia está presente en la literatura clásica, ${ }^{20}$ de lo que da muestra Ovidio (met. 2, 708-832) en la metamorfosis de Áglauro.

15 Sobre exempla $v$. Rodríguez Herrera (1997).

16 Litera Pythagorae discrimine secta bicorni/ Humanae uitae speciem praeferre uidetur,/ Nam uia uirtutis dextrum petit ardua callem,/ Difficilemque aditum primum spectantibus offert:/ Sed requiem praebet fessis in uertice summo./ Molle ostentat iter uia lata, sed ultima meta/ Praecipitat captos, uoluitque per ardua saxa./ Quisquis enim duros casus uirtutis amore/ Vicerit, ille sibi laudemque, decusque parebit./ At qui desidiam, luxumque sequetur inertem, / Dum fugit oppositos incauta mente labores, /Turpis, inopsque simul, miserabile transfiget aeuum. Todas las transcripciones de los poemas han sido realizadas por las autoras a partir de los florilegios en los que están insertos.

17 Sobre el simbolismo de la letra 'Y' $v$. Asencio (2004).

18 Liuor tabiscum malis uenenum, /Intactis uorat ossibus medullas./Et totum bibit artubus cruorem./ Quod quisque furit, inuidetque sorti,/Vt debet, sibi poena semper ipse est./ Testatur gemitu graueis dolores, /Suspirat, gemit, incutitque denteis./ Sudat frigidus intuens quod odit./ Effundit mala lingua uirus atrum./ Pallor terribilis genas colorat./ Infoelix macies renudat ossa./ Non lux, non cibus est suauis illi./ Non pottus iuuat, aut sapor Lyaei/ Nec si pocula Iuppiter propinet,/ Aut haec porrigat, et ministret Hebe,/ Aut tradat Ganymedes ipse nectar./ Non somnum capit, aut quiescit unquam./ Torquet uiscera carnifex cruentus. /Vesanos tacite mouet furores,/ Intentans animo faces Erynnis/ Letalis, Tityique uultur intus,/ Qui semper lacerat, commestque mentem./ Viuit pectore sub dolente uulnus,/ Quod Chironia nec manus leuarit,/ Nec Phoebus, sobolesue clara Phoebi.

19 Sobre los síntomas del amor, $v$. Traver (2011).

20 Una muestra de ello lo encontramos en los florilegios de Mirandula y Maior, quienes, bajo estos mismos lemas, extractan citas de Ausonio, Estacio, Horacio, Lucano, Lucrecio, Ovidio, Plauto, Propercio, Publilio Siro, Silio Itálico, Terencio y Tibulo. 
En cuanto al poema De Fortuna ${ }^{21}$ a diferencia de los anteriores, no critica el vicio ni elogia la virtud, sino que se ocupa de un tópico literario, la Fortuna mutabilis, ${ }^{22}$ incidiendo en su poder y en su carácter variable y arbitrario, pues eleva o humilla sin tener en cuenta los méritos. Los lemas de Maior y Mirandula coinciden en aludir a la inestabilidad de la Fortuna: Fortuna et eius inconstantia y De Fortuna, respectivamente; y el sublema Fortuna instabilis uersat hominum opera, secundum quosdam en Mirandula. La utilización de uersat nos sugiere el giro de la rueda de la Fortuna, tópico muy afín al de la Fortuna mutabilis.

De signis coelestibus ${ }^{23}$ en Maior lleva como lema Duodecim signa coelestia y aparece inserto entre poemas que consisten en enumeraciones o catálogos como Quatuor elementa, De mensibus et quatuor anni tempestatibus y De nominibus stellarum, Quatuor aetates hominum, De aetatibus animantium y Musarum inuenta. Estaba incluido en el apartado XI de los Carmina XII sapientum, dedicado a los signos del Zodíaco, que imponía como normas la utilización del hexámetro y la limitación a seis versos. Los poemas de enumeraciones,

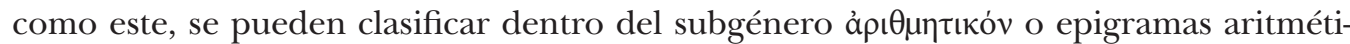
cos presentes en la Antología griega (Cairns 1973: pp. 15-22; Mondin 2016: pp. 198-199), en los que el número tiene un papel relevante ( 6 versos, 6 pies, 12 signos, es decir, 6+6). En Mirandula aparece bajo el lema De coelo y el sublema Coelestia figura quae? incide en la descripción astronómica.

Finalmente, nos encontramos con 3 poemas que aparecen solo en un florilegio: $D e$ Orpheo en los Illustrium poetarum flores y De aetatibus animantium y Musarum inuenta en las Sententiae ueterum poetarum.

Bajo el lema De carminibus y el sublema Carminum uis recoge Mirandula el poema De Orpheo.$^{24} \mathrm{El}$ héroe tracio, hijo del dios Apolo y de la musa Calíope, es considerado por la mitología griega el creador de la música. Con su lira es capaz de domeñar las fieras y conmover las piedras y los árboles; además, su música le permite entrar en las mansiones del Hades, apaciguar a sus habitantes y parar, por un instante, el castigo de los condenados (Ov. met. 10, 8-75). Ello hizo que, durante el periodo clásico y en la literatura

21 Fortuna potens, quam uariabilis,/ Tantum iuris atrox quae tibi uendicas,/ Euertisque bonos, erigis improbos,/ Nec seruare potes muneribus fidem./ Fortuna immeritos auget honoribus. /Fortuna innocuos cladibus afficit. /Iustos illa uiros pauperie grauat,/ Indignos eadem diuitiis beat./ Haec aufert iuuenes, et retinet senes,/ Iniusto arbitrio tempora diuidens./ Quod dignis adimit transfert ad impios,/ Nec discrimen habet, rectaque iudicat,/ Inconstans, fragilis, perfida, lubrica,/ Nec quos clarificat, perpetuo fouet,/ Nec quos deseruit, perpetuo premit.

22 La Fortuna en Roma puede entenderse como una diosa, similar a Tyche, a veces equivale a buena suerte y otras tiene carácter negativo, variable, ciega, etc. (Canter 1922: pp. 64-82). Del tópico fortuna mutabilis hay numerosas referencias en la literatura clásica. En los florilegios de nuestro corpus se ofrecen, sobre él, citas de Ausonio, Boecio, Horacio, Juvenal, Lucano, Manilio, Ovidio, Plauto, Séneca y Terencio. Sobre la rueda de la Fortuna $v$. Sánchez (2011).

23 Primus adest aries, Taurusque insignibus auro/ Cornibus, et Fratres, et Cancer aquatile signum,/ Tum Leo terribilis Nemees, atque innuba Virgo,/ Libra subit, caudaque animal quod dirigit ictum,/ Armatusque arcu Chiron, et corniger Hircus,/ Fusor aquae simul, et fulgenti lumine Pisces.

24 Threicius quondam uates fide creditur canora/ Mouisse sensus acrium ferarum,/ Atque amnes, tenuisse uagos,/ Et surda cantu concitasse saxa./ Suauisonosque modos testudines arbores secutae/ Vmbram feruntur praebuisse uati./ Sed placidis hominum dictis fera corda mitigauit,/ Doctaque uitam uoce temperauit./ Iustitiam docuit, coetu quoque congregauit uno,/ Moresque agrestes expoliuit Orpheus. Mirandula suprime los versos 7 y 8 del poema: Scilicet haud potuit, quae sunt sine, permouere, sensu/ (Finxere docti fabulam poetae). 
posterior, sobre todo en el Renacimiento, este exemplum, introducido por Virgilio (geor. 4, 453-527), sirviera de muestra del poder tranquilizador de la música (González Delgado 2012). Precisamente este carácter civilizador de la música es lo que interesa en este poema en el que el descenso de Orfeo al infierno es obviado a favor de la descripción del efecto de su canto en el bosque. Así lo ratifica el lema y el sublema con el que en este florilegio aparece.

Los dos poemas que solo aparecen en Sententiae ueterum poetarum no se encuentran en los Carmina XII sapientum y, como ya se ha indicado, se insertan entre una serie de poemas-catálogos muy del gusto de la epigramática helenística.

De aetatibus animantium ${ }^{25}$ es un epigrama aritmético en el que se nos proporcionan números con los que hay que operar para conocer las edades y ofrece un catálogo no cerrado. $^{26}$

En el poema Musarum inuenta, ${ }^{27}$ como es propio de los poemas enumerativos (Mondin 2016: pp. 198-199), se nombra a las nueve musas y se las describe brevemente en nueve hexámetros, pues, aunque el poema consta de once versos, los dos últimos se dedican a Apolo. El número nueve es la denominada “cifra plena”, al contener tres veces el número considerado perfecto, el tres. Neopitagóricos y neoplatónicos consideran que el hecho de que las musas sean nueve no es más que una referencia a este sentido de perfección (González Rodríguez 2011: p. 199). Al igual que Orfeo, las musas representan las artes que tanto gustan en el Renacimiento, pero además ambos, desde Platón, son considerados especialistas en el "encantamiento": mientras el uno embauca con su canto, las otras lo hacen a través de la inspiración.

En estos poemas hay dos aspectos muy diferentes que merecen nuestra atención, uno se refiere a la clasificación de Vir bonus y el otro a la cuestión de la autoría de los excerpta.

Respecto a Vir bonus, ya que se publica en algunas ediciones del Appendix, cabe preguntarse si ha de clasificarse como parte del Appendix o como una de las obras menores que circularon bajo el nombre de Epigrammata y fueron atribuidas falsamente a Virgilio. Todo apunta a que ediciones como la de Paruus han podido influir en los textos seleccionados, pues en ella se encuentran todas las obras que no aparecían en Carmina XII sapientum y Vir bonus se incluye entre los Epigrammata. Así pues, podríamos concluir que en Maior, como en Schönborn, no hay excerpta del Appendix, y considerar Vir bonus entre los Epigrammata. Decidir si Mirandula lo consideraba parte del Appendix o parte de los Epigrammata es un problema de difícil solución: los Epigrammata los ofrece completos y

25 Ter binos, decies nouem superexit in annos/ Iusta senescentum, quos implet uita uirorum./ Hos nouies superat uiuendo garrula cornix./ Et quater egreditur cornicis saecula ceruus./ Alipedum ceruum ter uincit coruus, at illum/ Multiplicat nouies phoenix reparabilis ales./Quem nos perpetuo decies praeuertimus aeuo,/ Nymphae hamadryades, quarum longissima uita est./ Haec cohibent fines uiuacia fata animantum./ Caetera secreti nouit deus arbiter uiae.

26 Aunque en muchos códices se atribuye a Virgilio, actualmente se adscribe a Ausonio, lo mismo que Vir bonus, De rosis nascentibus y Est et non (Alvar 1990: pp. 158-160; Moya 1982: p. 409; Roscher 1908).

27 Carmine Calliope libris Heroica mandat,/ Clio gesta canens transactis tempora reddit,/ Dulciloquis calamos Euterpe flatibus urget,/ Melpomene tragico proclamat moesta boatu./ Terpsichore affectus Cytharis mouet, imperat, auget./ Plectra gerens Erato saltat pede, carmine, uultu./ Signat cuncta manu, loquiturque Polyminia gestu./ Vraniaque poli motus scrutatur et astra./ Comica lasciuo gaudet sermone Thalia./ Mentis Apollineae uis has mouet undique Musas,/In medio residens complectitur omnia Phoebus. 
del Appendix solo fragmentos, pero ello puede deberse a su menor extensión y, además, el caso de Vir bonus en él es peculiar, ya que ofrece el poema fragmentado, pero completo (1-2; 3-13 y 14-26).

En cuanto a lo segundo, Mirandula y Schönborn señalan a Virgilio, sin duda ninguna, como autor de los poemas seleccionados. En cambio, Maior se muestra menos seguro y así, en De Fortuna y De Liuore, se limita a señalar Ex epigrammatibus quae Virgilio ascribuntur. Más escéptico aún es respecto a De uino et Venere, ya que indica Virgilius siue quisquis est autor, y a De littera Pythagorica, sobre la que con ligera variante también declara Virgilius aut quisquis est autor. De los cuatro restantes, Vir bonus, Duodecim signa coelestia, De aetatibus animantium y Musarum inuenta se limita a manifestar Incerti Autoris.

Además de los Epigrammata, hay otro texto atribuido a Virgilio que se recoge en uno solo de los florilegios de nuestro corpus, una breve composición de cuatro versos que aparece en los Versus sententiosi. Estos versos no presentan título ni referencia alguna a la obra virgiliana de la que proviene pues, según la tradición, remiten a una anécdota que se encuentra en la Vita Donatiana de Virgilio, en la que se relata que, enojado al saber que el poeta Batilo pretendía adjudicarse un texto de su autoría escrito en los muros de palacio, con el fin de demostrar quién mentía, Virgilio le propuso completar el texto sic uos, non uobis. Batilo fue incapaz de cumplir la tarea mientras que el de Mantua presentó los siguientes versos:

Sic uos, non uobis mellificatis apes:

Sic uos, non uobis fertis aratra boues:

Sic uos non uobis nidificatis aues.

Sic uos non uobis uellera fertis oues.

Aunque Schönborn los presenta en otro orden, ${ }^{28}$ recoge estos versos que se incluyeron a partir del siglo XV en los mss. de la vida donatiana de Virgilio. El hemistiquio Sic uos, non uobis ha gozado de gran rendimiento, ${ }^{29}$ es el lema del Cuerpo Facultativo de Archiveros, Bibliotecarios y Arqueólogos españoles desde su creación, hace más de 160 años (1858) (Carrión 2008: p. 38), y ha sido reutilizado para fines particulares, sobre todo, en escudos, emblemas y divisas. ${ }^{30}$

28 Sic uos, non uobis fertis aratra boues:/ Sic uos non uobis uellera fertis oues./ Sic uos, non uobis mellificatis apes:/ Sic uos non uobis nidificatis aues.

29 Una versión cristianizada de estos versos, bajo el título Pro franciscanis (s. XVIII), se encuentra en el Claustro del Convento de San Joaquín en Cieza (Murcia): Vellera fertis oves Francisci gregis egeni./ Fertis aratra boves tolerantes hispida Christi./ Nidificatis aves celeres ad celsa volantes./ Mellificatis apes Christi dulcedine pleni (Tudela 2009: p. 3). Otro ejemplo de reutilización es la obra de teatro Sic vos non vobis o la última limosna, del español José Echegaray (1832-1916), definida por él mismo como "Comedia rústica en tres actos y en prosa”. Igualmente lo encontramos en el himno Si madrugan los arqueros, canción compuesta por Gómez Molina (letra) y Cuesta Polo (música) para la Organización Juvenil Española (OJE), en una de cuyas estrofas aparece: "Mis compañeros salieron con el alba,/ sobre los arcos llevaban la canción: Sic vos non vobis, cantamos los arqueros/nuevas gargantas y un mismo corazón” (Jiménez 2005). Un estudio más detallado sobre esta anécdota y la fortuna de estos versos virgilianos puede leerse en Ruiz de Elvira (1989: pp. 33-38).

30 Entre otros, emplearon esta divisa el historiador segoviano Diego de Colmenares (1586-1651), el militar 


\section{Canto XIII de La Eneida de Maffeo Vegio}

En los Illustrium poetarum flores encontramos, además de lo ya expuesto, excerpta extraídos del libro XIII o Suplemento de La Eneida, una continuación de la epopeya virgiliana compuesta por el humanista Maffeo Vegio (Lodi, 1406/1407-Roma, 1458), cuya primera edición data de $1471 .{ }^{31}$ Como precedente de esta obra podemos citar los 89 hexámetros compuestos por Pier Candido Decembrio (1399-1477), Principium libri decimi tercii Aeneidos suffectum per P. Candidum adolescentem, que mostraban la muerte de Turno, el dolor de Latino y el funeral (Cristóbal 1993: p. 196). El Supplementum consta de 630 versos que contienen el funeral de Turno, el matrimonio de Eneas y Lavinia y finalmente la apoteosis de Eneas.

Los excerpta seleccionados son cuatro. El primero no es un texto narrativo, sino expresivo, con numerosas interrogativas retóricas y exclamativas que comprende los versos $143-157^{32}$ y que bajo el lema De cupiditate lamenta el deseo de dominio que conlleva numerosas muertes; el segundo extracto (vv. 279-283), ${ }^{33}$ con De morte como lema, es nuevamente un texto exclamativo en el que se interpela a la muerte señalando que es la única que frena las armas e iguala a todos; el tercer extracto (vv. 177-179), ${ }^{34}$ recogido bajo el lema De pulchritudine, consiste en una interpelación a Turno, ya muerto, y es una variante de ubi sunt? en el que se subraya que la muerte acaba con todo lo hermoso; en el cuarto extracto (349-351), ${ }^{35}$ que tiene De superbia como lema, se indica que a Turno no lo hicieron cambiar de propósito ni los ruegos ni las señales enviadas por los dioses.

A través de los sublemas se explicita el sentido de los textos seleccionados: Cupiditas regnandi malum exitum habet, el ansia de poder es nociva y conlleva muertes; Mors communis est omnibus, nullique parcit, solo la muerte a todos iguala; y Superbus precibus signisue a deo missis non flectitur, ut de Turno dicitur, el soberbio no cambia y ejemplo de ello es Turno. Frente al contenido moralizante de estos tres excerpta, el cuarto incide en el tópico literario de la fragilidad de la belleza: Pulchritudo bonum fragile est.

Antonio de Leyva, primer príncipe de Ascolti, o el poeta francés Barthélemy Aneau (López 2019).

31 Un acercamiento a la obra puede leerse en Chatillon (1984); Cristóbal (1993), entre otros. Brinton, en 1930, ofrece una reproducción de la princeps, reeditada en 2002 por Bristol Classical Press; Putnam \& Hankins (2004) reeditan y traducen el suplemento a partir de la edición que hiciera Schneider (1985) basada en la tradición manuscrita.

32 Quantos humana negocia motus/ Alternasque uices miscent? quo turbine fertur/Vita hominum? O fragilis damnosa superbia sceptri./ O furor, o nimium dominandi innata cupido,/ Mortales quo caeca uehis? quo gloria tantis/Inflatos transfers animos quaesita periclis?/ Quot tecum insidias? quot mortes? quanta malorum/ Magnorum tormenta geris? quot tela? quot enses/ Ante oculos si cernis habes? Heu dulce uenenum/ Et mundi letalis honos! heu tristia regni/ Munera, quae haud paruo constent: et grandia rerum/ Pondera, quae nunquam placidam promittere pacem/ Nec requiem conferre queunt heu sortis acerbae/ Et miserae regale decus magnoque timori/Suppositos regum casus, pacique negatos.

33 Heu mortem inuisam, quae sola ultricibus armis/ Elatos frenas animos, communia toti/ Genti sceptra tenens, aeternaque foedera seruans/ Quae magnos paruosque teris, quae fortibus aequas/Imbelles, populisque duces, seniumque iuuentae.

34 At nunc Turne iaces: ubi nam generosa iuuentae/ Gloria? Et excellens animus? quo splendidus altae/ Frontis honos? quonam illa decens tibi frontis imago?

35 Nec nostrae potuere preces inflectere durum,/ Nec diuum portenta animum, quin arctius ignem/ Spumebat ferus ore uomens, bellumque ciebat. 
No es inusual que, a partir de la editio princeps, tanto las traducciones a las lenguas vernáculas como las ediciones de La Eneida virgiliana incluyan el Supplementum de Vegio (Cristóbal 1993: p. 192). Un elemento más que ha podido influir en la inserción de fragmentos del Supplementum en los florilegios puede ser la tesis de Kallendorf (2005: pp. 173-212) de que Vegio hizo una lectura de La Eneida desde la perspectiva de la retórica epidíctica del elogio a Eneas y del vituperio a Turno. El final moralizante en el que no solo el soberbio sucumbe, sino que el piadoso es premiado hasta su apoteosis definitiva, justificaría, también, su presencia en los Illustrium poetarum flores.

\section{Conclusión}

En los florilegios estudiados se recogen extractos, atribuidos a Virgilio, que no corresponden a Eneida, Églogas y Geórgicas. Así, hay 17 fragmentos del Appendix pertenecientes a Aetna, Ciris, Dirae y Elegiae ad Maecenatem. Otros extractos se encuentran en los Carmina XII sapientum, actualmente atribuidos a Lactancio, pero que circularon bajo el nombre de Virgilio y se incluyeron en ediciones de este poeta bajo la denominación de Epigrammata. Son De duodecim signis, De uino et Venere, De Livore, De littera Pythagorica, De Fortuna y De Orpheo. No están en Carmina XII sapientum los poemas Vir bonus y De aetatibus animantium, atribuidos actualmente a Ausonio, y De Musarum inuentis, pero se publicaron bajo el título de Epigrammata junto al Appendix y a las tres grandes obras virgilianas. A ellos hay que añadir cuatro versos recogidos en la Vida de Virgilio de Donato y, por otro lado, cuatro fragmentos del Libri XII Aeneidos Supplementum compuesto por Maffeo Vegio.

La distribución de estos excerpta en los Florilegios es variable: solo hay fragmentos del Appendix y del Supplementum de Vegio, así como el poema De Orpheo, en Mirandula; únicamente Schönborn reproduce Sic uos, non uobis; y han sido seleccionados exclusivamente por Maior De Musarum inuentis y De aetatibus animantium. En los tres florilegios examinados aparece De uino et Venere y en Maior y Mirandula, Vir bonus, De duodecim signis, De Liuore, De littera Pythagorica y De Fortuna.

Actualmente, los poemas de los Epigrammata no se consideran obra del poeta augústeo y hay polémica, y una gran dosis de escepticismo, sobre la atribución a Virgilio de los del Appendix, pero solo Maior duda de la autoría de Virgilio del De Liuore, De littera Pythagorica, De Fortuna y De uino et Venere e, incluso, la niega para Vir bonus, Duodecim signa coelestia, De aetatibus animantium y Musarum inuenta.

La presencia en los florilegios de textos vinculados a Virgilio puede obedecer al gran prestigio del poeta de Mantua, al que se atribuían; pero, por otro lado, se refleja en ellos las dudas que sobre su autoría se habían suscitado.

En los textos seleccionados de De uino et Venere, Vir bonus, De Liuore, De littera Pythagorica y en los fragmentos de Vegio que aparecen bajo los lemas De cupiditate y De superbia, predomina el tono moralizador, la crítica de vicios, la alabanza de la virtud y la llamada a la moderación y a seguir el buen camino. El poema De Fortuna y los fragmentos del Supplementum ofrecidos bajo los lemas De morte y De pulchritudine recogen tópicos literarios 
como la fortuna mutabilis, la muerte igual para todos, la fragilidad de la belleza y ubi sunt? También se incluye en la selección un texto epidíctico, la alabanza a un héroe (De Orpheo), pero el elegido es un civilizador, que trae el arte de la música. Finalmente, sobre todo en Maior, se encuentran catálogos con una clara función pedagógica y mnemotécnica, es decir, intereses morales y didácticos pueden haber guiado la selección de estos textos.

En suma, los florilegios estudiados muestran claramente la difusión y conocimiento de estos textos espurios durante el siglo XVI. La auctoritas de Virgilio, que floreció durante la Edad Media, sigue presente en el Renacimiento, aunque en el caso de los textos estudiados con menor presencia frente a otros autores, especialmente Ovidio y Horacio. Por último, este estudio evidencia la complejidad de la tradición de los poemas menores de Virgilio y la relevancia pedagógica y literaria del Supplementum de Maffeo Vegio.

\section{Bibliografía}

Alvar Ezquerra, A. (Ed.). (1990). Ausonio. Obras (Vol. I). Madrid: Gredos.

Artigas, E. et al. (2014). De floribus florilegiisque barcinonensibus. In $\mathrm{M}^{\mathrm{a}} \mathrm{T}$. Callejas Berdonés et al. (Eds.), Manipulus studiorum en recuerdo de la profesora Ana María Aldama Roy (pp. 921-1056). Madrid: Escolar y Mayo.

Asencio González, E. (2004). La littera Pythagorica, el simbolismo de la 'y' en la literatura y en el arte. Alfinge, 16, 96-113.

Baehrens, E. (1881). Poetae latini minores (Vol. III). Leipzig: Teubner.

Brinton, A. C. (Ed.). (2002). Maphaeus Vegius and his Thirteenth Book of Aeneid (BCP classic commentaries on Latin and Greek texts). London: Bristol Classical Press.

Brummer, J. (1933). Vitae Vergilianae. Leipzig: Teubner.

Cairns, F. (1973). Catullus' Basia Poems (5, 7, 48). Mnemosyne, 26, 15-22.

Calderón Dorta, E. (1999). La embriaguez en Aristófanes: análisis léxico. Myrtia, 14, 5-18.

Canter, H. V. (1922). Fortuna in Latin Poetry. Studies in Philology, 19(1), 64-82.

Carrión, M. (2008). Del Cuerpo facultativo de archiveros, bibliotecarios y arqueólogos. In Sic vos non vobis: 150 años de Archiveros y Bibliotecarios (Catálogo de exposición; pp. 11-52). Madrid: Biblioteca Nacional y Ministerio de Cultura.

Chatillon, F. (1984). Sur Maffeo Vegio de Lodi, continuateur de Virgile au $\mathrm{XV}^{\mathrm{e}}$ siècle. Revue du Moyen Âge latin, 40(3-4), 213-217.

Cristóbal, V. (1993). Maffeo Vegio y su libro XIII de La Eneida. Cuadernos de Filología Clásica. Estudios Latinos, 5, 189-210.

Curbelo Tavío, Ma E., \& García de Paso, Ma D. (2020). La selección de los extractos virgilianos de La Eneida en los florilegios del siglo XVI. In G. Rodríguez Herrera (Ed.), Florilegios latinos y sociedad (pp. 29-67). Vigo: Academia del Hispanismo.

Fernández de la Cuesta, B. (2008). En la senda del Florilegium Gallicum. Edición y estudio del florilegio del manuscrito Córdoba, Archivo Capitular 150. Louvain-La-Neuve: Brepols.

Friedrich, A. (2002). Das Symposium der "XII sapientes". Kommentar und Verfasserfrage. Berlin: De Gruyter.

García Gual, C. (2003). Diccionario de mitos. Madrid: Siglo XXI de España Editores. 
García Soler, M. J. (2010). Mujer y vino en la comedia griega. In M. J. García Soler (Ed.), Expresiones del humor desde la Antigüedad hasta nuestros días (pp. 75-90). Vitoria-Gasteiz: Universidad del País Vasco.

González Delgado, R. (2012). Exempla y deuda literaria: los suplicios infernales en los descensus ad inferos de la literatura grecolatina. In M. L. Harto Trujillo, \& J. Villalba Álvarez (Eds.), Exempla fidem faciunt (pp. 63-74). Madrid: Ediciones clásicas.

González Rodríguez, A. M. (2011). Las Musas y la Inspiración del poeta: a propósito de «El Parnaso» de Nicolas Poussin del Museo del Prado. Anales de Historia del arte, 11, 193-230.

Green, L. D., \& Murphy, J. J. (Eds.). (2006). Renaissance rhetoric short-title catalogue 1460-1700. Aldershot, UK - Burlington, USA: Ashgate.

Jiménez Calvente, I. (2005). Si madrugan los arqueros: un estudio sobre socialización política a finales del franquismo. Granada: Port Royal Ediciones.

Kallendorf, C. (2005). Elogio de Eneas. Virgilio y la Retórica Epideíctica en el Temprano Renacimiento Italiano. Santiago de Chile: Ril Editores.

Kallendorf, C. (2013). Virgil in the Renaissance Classroom: From Toscanella's Osservationi [...] sopra l'opere di Virgilio to the Exercitationes rhetoricae. In J. F. Ruys, J. Ward, \& M. Heyworth (Eds.), The Classics in the Medieval and Renaissance Classroom: The Role of Ancient Texts in the Arts Curriculum as Revealed by Surviving Manuscripts and Early Printed Books (pp. 309-328). Turnhout: Brepols.

Kallendorf, C. (2018). Canon, print, and the Virgilian corpus. Classical Receptions Journal, 10 (2), 149-169.

López Poza, S. (2019). SIC VOS NON VOBIS. In Symbola: divisas o empresas históricas. BIDISO Biblioteca Digital Siglo de Oro [retrieved 01.10.2020 from https://www.bidiso.es/Symbola/divisa/247].

Mondin, L. (2016). Talia in cattedra: usi didascalici dell'epigramma tardolatino. In L. Cristante, \& V. Veronesi (Eds.), Forme di accesso al sapere in eta tardoantica e altomedievale (Vol. VI; pp. 189-235). Trieste: Edizioni Università di Trieste.

Mondin, L. (2019). The Late Latin Literary Epigram (Third to Fifth Centuries CE). In Ch. Henriksen (Ed.), A Companion to Ancient Epigram (pp. 577-595). Hoboken: Wiley.

Moreno Soldevila, R. (Ed.) (2011). Diccionario de motivos amatorios en la literatura latina (siglos III a. C. $-I I$ d. C.). Huelva: Universidad de Huelva.

Moya del Baño, F. (1982). Virgilio y la Appendix Vergiliana. Helmantica: Revista de filología clásica y hebrea, 33 (101-102), 407-448.

Olsen, B. M. (1979; 1980). Les classiques latins dans les florilèges médiévaux antérieurs au XIII ${ }^{\mathrm{e}}$ siècle. Revue d'histoire des textes, 9, 47-121; 10, 47-172.

Putnam, M. C. J., \& Hankins, J. (Eds.). (2004). Maffeo Vegio: Short epics. Cambridge, Mss. - London: Harvard University Press.

Recio García, T. A., \& Soler Ruiz, A. (1990). Introducción al Apéndice Virgiliano. In Iidem (Eds.), P. Virgilio Marón Bucólicas. Geórgicas. Apéndice Virgiliano (pp. 397-421). Madrid: Gredos.

Rodríguez Herrera, G. (1997). Exempla mythologica en las tragedias de Séneca. In M. Rodríguez-Pantoja (Ed.), Séneca dos mil años después (pp. 211-221). Córdoba: Servicio de Publicaciones de la Universidad de Córdoba.

Rodríguez Herrera, G., Rodríguez Rodríguez, G., \& Santana Jaria, O. (2020). El estudio de los 
florilegios latinos del siglo XVI en la era digital: el Proyecto Excerpta. In J. Ma. Maestre Maestre et al. (Eds.), Humanismo y pervivencia del mundo clásico VI. Homenaje al profesor Eustaquio Sánchez Salor. Alcañiz - Madrid: CSIC.

Roscher, W. H. (1908). Zu Ausonius de aetatibus animantium. Philologus, 67, 158-160 [retrieved 01.10.2020 from https://doi.org/10.1524/phil.1908.67.14.158].

Ruiz de Elvira, A. (1989). Sic vos non vobis. Cuadernos de filología clásica, 22, 33-38.

Sánchez Márquez, C. (2011). Fortuna velut luna: iconografía de la 'Rueda de la Fortuna' en la Edad Media y el Renacimiento. EHumanista, 17, 230-253.

Schniebs, A. et al. (Eds.). (2014). Copa. La tabernera: poema pseudovirgiliano. Ciudad Autónoma de Buenos Aires: Editorial de la Facultad de Filosofía y Letras Universidad de Buenos Aires.

Thiercy, P. (1997). Le palais d'Aristophane ou les saveurs de la Polis. In P. Thiercy, \& M. Menu (Eds.), Aristophane: La langue, la scène, la cité. Actes du colloque de Toulouse, 17-19 mars 1994 (pp. 131-177). Bari: Levante editori.

Traver Vera, Á. J. (2011). Síntomas de amor. In R. Moreno Soldevila (Ed.), Diccionario de motivos amatorios en la literatura latina (siglos III a. C.-II d. C.) (pp. 398-402). Huelva: Universidad de Huelva.

Tudela, J. L. (2009). VELLERA FERTIS. Reflexiones de un lector de Virgilio sobre los versos del Convento. Andelma, Boletín Informativo del Centro de Estudios Históricos Fray Pasqual Salmerón, 18, 3-7.

Dra. Ma Elena Curbelo Tavío / mariaelena.curbelotavio@ulpgc.es

Research Institute of Text Analysis and Applications (IATEXT)

University of Las Palmas de Gran Canaria

Campus del Obelisco (Aulario del Obelisco, módulo A)

Pza. de la Constitución, s/n. 35003 Las Palmas de Gran Canaria, Spain

Dra. Ma Dolores García de Paso Carrasco / mdolores.garciadepaso@ulpgc.es

Research Institute of Text Analysis and Applications (IATEXT)

University of Las Palmas de Gran Canaria

Campus del Obelisco (Aulario del Obelisco, módulo A)

Pza. de la Constitución, s/n. 35003 Las Palmas de Gran Canaria, Spain

This work can be used in accordance with the Creative Commons BY-SA 4.0 International license terms and conditions (https://creativecommons.org/licenses/by-sa/4.0/legalcode). This does not apply to works or elements (such as image or photographs) that are used in the work under a contractual license or exception or limitation to relevant rights. 